\title{
Reagent Lot Number
}

National Cancer Institute

\section{Source}

National Cancer Institute. Reagent Lot Number. NCI Thesaurus. Code C112406.

The lot number of the reagent. 